\title{
El nuevo paradigma de la complejidad y la educación: una mirada histórica*
}

\author{
Taeli Gómez Francisco**
}

Resumen: La educación, como proceso de formación de los seres humanos para la sociedad, se encuentra determinada por los requerimientos del ser social. Ésta se valida toda vez que mantiene coherencia con los paradigmas, categorías, o las bases epistemológicas de un determinado cuadro científico de mundo o imagen de éste. De acuerdo a ello, develamos que las crisis o problemas que tiene el proceso educacional actual, obedecen a un movimiento objetivo de tensiones dadas por las necesidades sociales y los obstáculos de las micro y macro relaciones de poder sustentadas en paradigmas clásicos.

Palabras clave: práctica social, cuadro científico de mundo, paradigma clásico, complejidad, educación

\section{The new paradigm of complexity and education: an historical glance}

\begin{abstract}
: education as a process of formation for human beings insert in society it is determined by the requests of the social being. It is validated as much as it is coherent with the paradigms, categories or the epistemologycal bases of a specific scientific world vision or an image of it. Acording to that, we unfold that the crises or problems the current educational process has, is due to a specific tensional movement produced by social needs and by the obstacles of micro and macro power confrontations upholded by classical paradigms.

Key words: social practice, epistemologycal bases of scientific world vision, classical paradigm, complexity, education
\end{abstract}

\section{O novo paradigma da complejidad e a educação: uma mirada histórica}

Resumo: A educação, como processo de formação dos seres humanos para a sociedade, se encontra determinada pelos requerimientos do ser social. Esta se valida toda vez que mantém coerência com os paradigmas, categorias, ou as bases epistemológicas de um determinado quadro científico de mundo ou imagem deste.

\footnotetext{
* Este artículo contiene investigaciones comenzadas con la Tesina (2003) realizada para el Diplomado impartido por la Universidad de Ciego de Ávila, Cuba en Chile; asimismo, la experiencia como docente, principalmente, como autora de la Cátedra de Complejidad y Derecho impartida en la Universidad de Atacama, Chile.

** Universidad de Atacama, Copiapó, Chile. Email: taelig@yahoo.es.; taeli.gomez@uda.cl
} 
De acordo a isso, develamos que as crises ou problemas que tem o processo educacional actual, obedecem a um movimento objectivo de tensões dadas pelas necessidades sociais e os obstáculos das micro e macro relações de poder sustentadas em paradigmas clássicos.

Palavras-chave: Prática social, quadro científico de mundo, paradigma clássico, complexo, educação

Recibido: 18.01.2010

Aceptado: 07.02.2010

$* * *$

\section{Una introducción a tener en cuenta}

El nuevo siglo se ve enfrentado a enormes desafíos que vienen desde sociedades, cada vez más interrelacionadas universalmente. Así, es posible observar cambios económicos significativos de mundialización de capitales y mercados, tanto industriales como financieros; avances extremadamente, dinámicos, en la ciencia y tecnología, en esferas mecánicas, virtuales, espaciales, etc. Todo lo cual, ha afectado a los humanos - y no humanos- que vivimos esta realidad. Nuestras culturas han absorbido todos estos nuevos aspectos mundializados, haciéndolos propios e intercambiables. Las comunicaciones, sin importar la lejanía de los lugares, son casi instantáneas, en tiempo real; la movilidad de información es sorprendente; las guerras televisadas, el conocimiento de otras formas de vida, de invasiones, dolores y relaciones entre países y personas de los más variados e insospechados lugares, representan nuestro cotidiano. La alfabetización se vincula con el computador; la realidad virtual es casi una nueva compañía de los niños, más cercana que el trompo, las canicas o los juegos de creación manual y colectiva.

Estamos sin duda, inmersos en una revolución científico-técnica que significa una nueva forma de producir y pensar realidad. Las necesidades y problemas teórico-prácticos, han demandado cambios y rupturas epistemológicas, e incluso, de la propia racionalidad. A denominación de Thomas Kuhn, podría decirse que estamos frente a cambios de paradigmas, como resultantes de revoluciones científicas.

Se puede ilustrar la afirmación anterior, con la demostración que nos permiten las distintas disciplinas; comenzando con la física que, desde la relatividad, los nuevos significados del tiempo y espacio y la física cuántica, han dado lugar a una de las importantes rupturas para la noción de realidad, entre otras, las nuevas perspectivas sobre la incertidumbre y la relación entre el sujeto y objeto de conocimiento, planteado por Heisenberg. O bien, las nuevas perspectivas de las estructuras disipativas de Ilya, Prigogine; las investigaciones de Humberto R. Maturana y Francisco J. Varela sobre la autopoiesis y su extrapolación -de ésta- a las ciencias sociales, realizadas por Luhman. Las nuevas dimensiones del planeta que amplían la denominación de biosfera propuesta por el geólogo ruso Vladimir I. Vernadsky, como la Hipótesis Gaia, dada por James Lovelock y Lynn 
Margulis; asimismo, la antropología con R. Rappaport y Vayda, dan paso a la unificación e interrelación entre cultura y medio ambiente en interdependendencia. No se puede dejar de mencionar a la generalizada Teoría General de Sistemas propuesta por Ludwig Von Bertalanffy; como tampoco, los significativos aportes de la Teoría Cibernética de Winer y las propuestas de la Cibernética de Segundo Orden de Foerster; los cambios necesarios de las matemáticas para dar cuenta de un objeto real-complejo, con las Teorías del Caos, de las Bifurcaciones y Catástrofes, y la Teoría de los Fractales; sólo por mencionar algunas.

A finales del siglo XX, estos movimientos científicos parciales y disciplinarios, no sólo comenzaron a interrelacionarse, sino que, además, a dimensionarse -con el aporte de la Filosofía como saber omnicomprensivo, como un movimiento único ${ }^{1}$. En este sentido, algunos autores comenzaron a identificar y masificar profundas reflexiones sobre las semejanzas sincrónicas, a pesar, de las distintas parcelas y problemas del conocimiento. Todo lo cual, ha dado como resultado la configuración y denominación de un nuevo paradigma científico generalizador, capaz de abarcar a todas las ciencias, como lo es el surgimiento de este nuevo paradigma complejo o de la complejidad(es) ${ }^{2}$

Detallaremos este nuevo paradigma - el complejo-, a través, de la adopción de algunas líneas de ruptura con el paradigma clásico ${ }^{3}$. Entre los autores que se han destacado por investigarlas a fondo, encontramos a Delgado (2004), que menciona cinco, como las más importantes, a) el cuestionamiento con el ideal cartesiano de separación absoluta entre el sujeto y el objeto del conocimiento; b) una nueva noción de realidad, la cual, comienza a ser concebida como proceso, mas no como una entidad final "hecha”, "acabada”, “dada”; c) la reconsideración del determinismo, causalidad, predicción y el cambio en la forma y el ideal de lo que estas nociones presuponen; d) el reconocimiento de los valores como integrantes de la cognición humana con la intención de superar la separación absoluta entre el conocimiento y los valores e) y en consecuencia, con las ideas anteriores, la demanda de responsabilidad como elemento constitutivo de la producción de conocimientos científicos. Estas líneas de ruptura -como

\footnotetext{
${ }^{1}$ Entre autores que han trabajado con mayor reflexión abarcadora, desde finales del siglo XX podemos citar a Fritjof Capra, Edgar Morin, Carlos Jesús Delgado, Thalía Fung, Pedro Luis Sotolongo; Carlos Maldonado, Asimismo existen instituciones como, el Center for the Study of Complex Systems (CSCS) como Programa Interdisciplinario de la Universidad de Michigan, la Association pour la Pensée Complex fundada por Edgar Morin, para favorecer un pensamiento transdisciplinario, la Cátedra itinerante de la UNESCO Edgar Morin dedicado a la formación en Pensamiento Complejo y Epistemología Compleja, la Cátedra para el estudio de la complejidad propiciado por el Instituto de Filosofía de La Habana de Cuba, dedicado a temas relacionados con la complejidad y sus implicaciones teóricas, epistemológicas y metodológicas, sólo a modo de ilustración.

${ }^{2}$ Utilizaremos indistintamente ambas posibilidades, en tanto, no es posible afirmar sólo un tipo de complejidad; principalmente, por la emergencia que señala este paradigma.

${ }^{3}$ Nos referiremos al paradigma clásico de manera general, en contraposición al complejo.
} 
las denomina el autor citado-, dan orientación, a la vez, sobre los elementos conformadores del paradigma científico clásico y el nuevo complejo.

Este paradigma científico, viene a superar la insuficiencia histórica, del paradigma clásico y su correspondiente valoración a la noción de simplicidad y dominación del hombre hacia la naturaleza (Bacon, 1998). Supera, por lo tanto, la identificación de la complejidad con algo complicado y por el contrario, la trascendencia consiste, en afirmar que lo complejo es un atributo de la realidad y que ésta es, por lo tanto, irreducible a entidades discretas. Sus propuestas valoran las unidades dialécticas de lo simple y lo complejo, la validación del azar, la incertidumbre, el caos, la indeterminación y emergencia, lo no lineal, etc.

Ahora bien, este paradigma de la complejidad, no sólo viene a conformarse desde lo ontológico de cómo es la realidad, sino que, requiere de una coherencia epistemológica, de cómo se piensa y concibe para dar sentido a este atributo de la sociedad y naturaleza que rebasa lo simple ${ }^{4}$. De ahí que, las propuestas de la epistemología de segundo orden, el pensamiento complejo o la racionalidad no clásica, son cada vez más admitidas.

En tal sentido, también, los nuevos diálogos de saberes científicos, provienen del reconocimiento de las incapacidades de las obstinadas autonomías disciplinarias, para dar respuesta a los nuevos requerimientos de las complejidades del mundo. No menor, es la prueba proveniente de los nuevos enfoques metodológicos holistas. Objetivadas en las direcciones, no sólo multi o interdisciplinar; sino que, las simbiosis entre nuevos y, hasta entonces, ocultos o inexistentes objetos de estudios, han dado paso a la transdisciplina y perspectivas heurísticas. Bien describe Delgado (1999: 67) "El objeto de la cognición está dado en forma de práctica, y nuestra idea del mundo es totalmente dependiente de ella: vemos en el mundo, lo que nuestra práctica nos permite ver. De modo que la idea de mundo,- de cómo es, qué objetos lo integran, cuál es su orden, o si existe o no- esta en dependencia directa de nuestra práctica"

En definitiva, las necesidades del mundo en su dinámica económica, social y política, como la magnitud de la crisis ecosocial, ha demostrado la insuficiencia de los paradigmas simplistas y reduccionistas para dar soluciones a los cambios requeridos por éste; de ahí que, la Filosofía y su enfoque omnicomprensivo, ha colaborado en identificar e impulsar el nuevo paradigma científico complejo o de la complejidad.

Ahora bien, resulta imperativo explicitar que este proceso, tanto de conformación de un paradigma, como de rupturas con los antiguos, está vinculado, con la complejidad del mundo, sus interrelaciones económicas y la universalización del trabajo; por consiguiente, con necesidades materiales que requieren, de las ciencias, nuevas respuestas y soluciones. Por lo

${ }^{4}$ Maldonado (1999), señala a la complejidad como ciencia, método y cosmovisión. 
mismo, conforman un movimiento histórico, son reflejos de éste y no una elección subjetiva.

En el contexto histórico-epistemológico de cambios de paradigmas, antes expuesto, nos surge la inquietud, ¿Qué sucede con la educación?

De acuerdo a lo anterior, nos surge otra interrogante; si, a pesar, de todos los cambios producidos a nivel de nuestra realidad -social, económica, cultural, política, ambiental- y de su noción espiritual - entendida como los sistemas categoriales y los nuevos modelos que la explican, principalmente, desde el paradigma de complejo- ¿Por qué la educación sigue reproduciendo paradigmas clásicos reduccionistas, con la generación de problemas sin solución bajo tales referentes? De ahí, que una de las hipótesis de trabajo principal se puede describir de la siguiente forma: Al incorporar de manera consciente el nuevo paradigma de la complejidad al proceso educativo docente se dilucidan problemas, interrelaciones y posibles objetos de estudio nuevos, que hasta ahora, no se han solucionado ni observado.

Para ser coherentes con la propuesta metodológica, abordaremos el trabajo, desde una metodología heurística-dialéctica. Esta orienta el movimiento que valora la correlación del todo y la parte de manera dialéctica, relacional con esfuerzo por superar perspectivas enfocadas como unidades discretas. El movimiento va desde el todo (comprensión) a las partes (explicación) y desde éstas al todo integrado (interpretación). Estos tres procesos comprenden una unidad dialéctica que permite avanzar en el conocimiento un proceso educativo nuevo.

\section{Desde el todo (comprensión): La educación como proceso contextualizado}

\section{La educación en el contexto de un paradigma clásico}

En sus orígenes, el capitalismo, como nueva formación económicosocial, lo revolucionó todo; las formas de producir, pensar y sentir realidad. De acuerdo a esto, el proceso de formación o educación formal e informal de los sujetos que la autorreproducirían, es afectado, en la misma medida, que el resto de las circunstancias; pues participan de las mismas modificaciones del cuadro científico de mundo. Es decir, la educación será determinante para la forma que los sujetos, van a comprender el mundo e interactuar en él, consigo como sociedad y naturaleza.

Ahora bien, esta formación económica social se reproduce idealmente a si misma, tiene su imagen del mundo y de la realidad, a tal representación se le denominado cuadro científico clásico de mundo. Éste significa la idea de mundo, sobre la cual se va a actuar, pensar y conocer; su imagen afecta la teoría y empíria; por lo tanto, delimita la forma, el quién, el qué y cómo se conoce. Este se conforma de acuerdo a los procesos que sintetizan todos los reflejos ideales. De ahí, que el cuadro científico clási- 
co de mundo, se estableció, con aportes de científicos, filósofos, políticos; con los elementos que bien destaca y describe Kedrov, a) la idea de inmutabilidad de la naturaleza, b) la comprensión de los átomos como partículas últimas-ladrillos del universo-indivisibles e inmutables de las que todo está hecho, c) la evidencia mecánica, d) la suposición de que el mundo es dado. Estos cuatro ideales sentaron las bases de una comprensión científica que devino dominante desde el siglo XVII hasta el siglo XIX, y que extiende su influencia hasta nuestros días (Delgado, 1999: 69).

En el contexto de un cuadro científico clásico de mundo se conforman modelos científicos explicativos como el paradigma científico clásico, éste comprende la simplicidad, la dualidad cartesiana; una noción de realidad, como entidad final "hecha”, “acabada”, “dada”; sobre la base del determinismo, causalidad, predicción. Todo logrado, a través, de un método, un camino que acerca al sujeto a la verdad y objetividad del objeto de conocimiento; con todos sus procedimientos y fundamentos filosóficos que lo validaron de la misma forma que, lo hicieron con su producto; de ahí la hegemonía del saber científico sobre otros.

Este paradigma científico clásico fue recepcionado por la psicología -más bien la posesionan como ciencia- y por ende, por los modelos educativos. Así por ejemplo, el modelo Conductista, se define por poner la atención en la conducta humana observable y no en la conciencia. (Se observa como un hecho más de la naturaleza, con la misma reducción analítica, causalista, desconectada de los procesos generales, y predecible, bajo la construcción de un sujeto, como bien ésta última reflexión la trabaja Pérez (1996). Se basa en la existencia condicionada de que, a un estímulo, le sigue una respuesta, como resultado de la interacción entre el organismo que recibe el estimulo y el medio ambiente, como un otro. Lo podemos identificar dentro de un enfoque clásico de Pavlov y Skinner; es decir, plenamente coherente con el sentido del pensamiento científico. Dentro de la misma lógica los aborda Lipman (1998) que, al estudiar el pensamiento complejo, crítico, detalla un modelo educativo determinado por lo que él señala paradigma estándar que limitan o reducen esta reflexión, como aquellos que consideran que la educación consiste en la transmisión de conocimiento, de quienes saben, a los que no; sobre un mundo no misterioso; el conocimiento, además, se distribuye en disciplinas, las que no se sobreponen y juntas, conforman el conocimiento total del mundo; asimismo, el profesor juega un papel autoritario, pues quiere que los estudiantes conozcan lo que él conoce y el conocimiento, en definitiva, lo adquieren mediante la absorción de datos e información.

Estos modelos, sean los denominados conductistas, o clásicos, o basados en paradigmas estándar, en rigor, participan de lo que hemos denominado paradigma científico clásico y por ende, dentro de la lógica de un cuadro científico clásico de mundo. Ello, porque en definitiva, se encuentran en el mismo concreto histórico dado por una específica formación económico-social; la cual, requiere que los sujetos sean formados para los 
requerimientos de su necesidad. Por lo tanto, sólo se puede aplicar en el contexto de una forma cultural específica, le pertenecen a ella y tienen sentido.

Para detallar lo anterior, del cómo los modelos educacionales son coincidentes con el paradigma científico clásico y cuadro científico clásico de mundo, diremos:

En un primer aspecto, desde una perspectiva epistemológica, el proceso educacional parte desde la validación histórica de un conocimiento que hay que transmitir, pues está acabado. En rigor, el profesor sabe (culturalmente hablando), que la naturaleza es inmutable y su movimiento es mecánico y que, al estar conformada de ladrillos newtonianos, sean átomos, personas, células o entidades discretas, se puede, sobre ellos, establecer relaciones causales e identificaciones transmisibles. Además, el conocimiento de lo anterior, es el producto de un proceso dado sobre la base de la dualidad de un sujeto que está fuera del objeto de conocimiento, y si este último es inmutable, regular, se puede, a través de los métodos científicos, conocer, medir, predecir. Porque la formación del conocimiento, en definitiva, es acabado y hay que entregarlo de esa forma. De ahí que resulte adecuado, un diseño educacional y pedagógico, que lo reproduzca de esa forma (en este sentido, hay que reconocer que históricamente fu acertado, en su lógica, sólo que ahora, la ha perdido; porque el mundo ya no es tan sólo mecánico, sino que lo es interrelacionadamente, como también financiero). El aprendizaje parte de la idea de un sujeto pasivo que recibe ese conocimiento, como un recipiente que memoriza, porque el conocimiento está como un objeto, fuera de él.

En un segundo aspecto, la actividad pedagógica, se entiende como un momento científico, como cualquier otro, al igual que un químico frente a su microscopio. Éste debe mirar a un alumno como un objeto de investigación y manipulación, al que hay que observar, predecir y cuantificar. La evaluación del resultado se hace, a través, de la dosificación de la asimilación a la entrega de un conocimiento estático que fue adquirido de la misma forma por el profesor, de acuerdo a la lógica del primer aspecto. Además, el lugar apropiado es la escuela porque el proceso es divisible e identificado.

En definitiva, estos elementos no estaban errados o equivocados, constituyeron el reflejo de un momento histórico, obedecieron a las necesidades de una formación económico-social, la misma que produjo un cuadro científico clásico de mundo y los paradigmas acordes a su movimiento. Por lo tanto, en y desde el cual, se validaron porque permitieron formar a los humanos que reproduciría tal devenir.

Ello explica la existencia histórica de modelos educacionales, como igualmente, permite comprender las contracciones y tensiones que ocasiona la aplicación de un modelo que se declara, como el constructivista y que, producto de las micro y macro relaciones de poder, no se aplican y sólo lo hacen, como un conductismo encubierto con didácticas. En síntesis, se re- 
produce la misma contradicción social, por un lado, las necesidades del ser social producto del avance de las fuerzas productivas y el obstáculo que ocasionan las relaciones de poder.

\section{La educación en el contexto de un paradigma no clásico}

En los últimos tiempos han existido cambios que han modificado nuestras actuaciones, no obstante, se mantiene la esencia de la relación contradictoria capital-trabajo. Efectivamente, ya no se producen ganancias necesariamente, sobre la base de la producción industrial, sino que la acumulación de ésta, ha producido mercados financieros que actúan, tan anárquica y caóticamente como la dinámica de las mercancías; pero de igual forma, hay que reconocer la novedad y complejidad de las dinámicas aceleradas, con un mayor grado de sensibilidad a los cambios de las condiciones iniciales; ello ha dado lugar al surgimiento de fuerzas productivas no pensadas, tanto mecánicas como virtuales. Hay que señalar, del mismo modo, que las crisis son cada vez más universales, abarcando ahora la dimensión de ecosocial.

Ante tales requerimientos del ser ecosocial -objetivo- ya se han sucedido cambios en el cuadro científico de mundo irreversibles, gracias a las revoluciones científicas que han incorporado aspectos que fueron terminando con el clásico y que sostuvo al conductismo y/o a los modelos educativos tradicionales como práctica, -no sólo como idea-.

Como bien sintetiza Kedrov, (1990) en sus estudios, se han identificado grandes revoluciones que han modificado profunda e irreversiblemente el cuadro científico clásico de mundo como la "copernicana”, "kantiana”, "cuántico-relativista” y "científico técnica”; la primera, provocó un cambio a la elevación del pensamiento abstracto por sobre el común (es la tierra la que gira alrededor del sol, a pesar que ello no se ve); la kantiana dio un paso hacía el quiebre de la inmutabilidad de la naturaleza, por la idea de cambio; pero ya sirvió está a la cuántico-relativista, en la que se comenzó por terminar con la noción atomista de la conformación de un mundo compuestos por átomos indivisibles desplazando a la evidencia mecánica; pero la científico- técnica ya en la segunda mitad del siglo XX, ha dejado agónico al cuadro clásico de mundo, para dar paso a uno que se configura sobre una concepción de la realidad como una totalidad compleja, con algunos aspectos que se han podido sistematizar, relacionados con los ya explicitados anteriormente en :

- Una nueva valoración a lo complejo, pues en el clásico, se asociaba con algo negativo y complicado. La complejidad, eran barreras que había que despejar, hasta reducir.

- La idea de lo simple, de la evidencia mecánica, la composición de mundo por átomos-ladrillos, ha perdido sentido histórico; la propia física aporta al cambio que, a pesar de llegar 
al quark, en su lógica, hace desaparecer el sentido de lo divisible bajo el posicionamiento de entidades difusas, imposibles de aislar.

- Hoy la realidad no puede entenderse sino como una multiplicidad de relaciones que la conforman, y por lo cual, todo se encuentra interconectado y su separación es una ficción.

- Hay una nueva relación entre el todo y las partes.

- Lo complejo como atributo le pertenece, es inherente a la realidad, y afirma que los sistemas de la naturaleza, no son dados de antemano, ni inmutable; tanto que se reconoce que los sistemas pueden variar totalmente si se cambian en mínimo las condiciones iniciales. De ahí la dificultad de propiciar la dualidad de ciencias duras y blandas o naturales y del espíritu.

- Ya no es posible seguir con la dualidad cartesiana de sujetos y objetos separados, están interrelacionados. Como también la propia consideración del sujeto ya no es considerado como un átomo, sino como un sistema.

- Y por consiguiente, para esta realidad compleja el único pensamiento que la puede entender, comprender y formular, es el pensamiento dialéctico, hermenéutico, contextuado y complejo. El mundo simple ya no es real, es una categoría que no la expresa. La verdad, y el conocimiento van a depender de las relaciones que emergen de las interacciones, no está dado, por eso no se puede entregar, transmitir como conocimiento, hay que construirlo desde la práctica (ello hace que la mayor coherencia histórica-interna con un modelo pedagógico, sea con propuestas más constructivistas, críticas y relacionales).

- Tampoco se puede reducir la comprensión de la realidad a esferas aisladas disciplinariamente, de ahí su enseñanza en especialidades, como la física, matemáticas, ciencias sociales etc., en la que, cada una de ellas, enseñaba parte del conocimiento. Hoy debe ser superado por uno práctico-holista.

- De manera interesada, diremos que la determinación material y dialéctica se valida.

Como se aprecia, no es posible utilizar categorías de un mundo, que ya no existe, pues ello, no expresaría más, que un autoritarismo dogmático, un desfase con la práctica. Y muy grave resultaría, que la educación, como proceso general de formación de humanos para el desarrollo social, esté hoy presentando, una realidad que no existe, convirtiéndose en un multiplicador de alienación. 


\section{Las partes (explicación): \\ Educación, y paradigma científico clásico y complejo}

Difícil resulta, desde una perspectiva práctica, reconocer la existencia de los elementos conformadores del paradigma clásico, por consiguiente, reproducirlas como base sustentadora de los modelos educacionales y pedagógicos. Veremos algunas ilustraciones.

1. La presencia de la dualidad sujeto/objeto de conocimiento, provoca distintas esferas problemáticas; a) la reproducción que hace el profesor de ellas, al creer recibir el conocimiento obtenido por un sujeto y entregarlo como objeto sagrado al educando; b) la suposición y por ende, manipulación que el educador hace del objeto-estudiante que, al concebirlo de esa manera, se transforma en un objeto más observable y manipulable, se aplican en él todas las consideraciones causalistas reduccionistas etc., y cuando este quiere demostrar su integralidad como ser emergente, se etiqueta como un ser rebelde, indisciplinado y marginal. c) la relación entre el sujeto y el objeto de la cognición, separa "todo"; al alumno del mundo, al aula de la calle; a lo cognitivo de lo emocional y lo valórico; al sujeto histórico de su esencia; la organización de profesores y alumnos; la evaluación en la prueba de lo que se vive en la sociedad, de ahí la incoherencia que el alumno se puede sacar una nota máxima en una evaluación sobre la teoría de los valores y ser un humano egoísta.

2. El reconocimiento de la linealidad, simplicidad, orden y certidumbre, impide al educando la creación y la comprensión del mundo que es contrariamente a ello, complejo con aleatorios momentos simples.

3. La validación que aún hace el directivo- docente de la realidad concebida bajo el sentido de un paradigma científico clásico - esto es basada en la lógica de la dualidad sujeto y objeto del conocimiento; la noción de realidad, como una entidad final "hecha", "acabada", "dada"; determinista, bajo la lógica causalidad y de la predicción; y la separación entre cognición y valor, por lo tanto, en la creencia de la objetividad y neutralidad del conocimiento- le impide, entender la profundidad de modelos educacionales complejos. Es decir, hay una dicotomía entre la cosmovisión -clásica- y los nuevos modelos educativos y pedagógicos. Por ello, es lógico que, modelos como el constructivista, sean entendidos, sólo en la dimensión metodológica como didácticas.

En algún sentido, se puede llegar a observar, la existencia de un gran esfuerzo en materiales e inventivas para aplicarlo, pero en rigor, para no aplicarlo; en última instancia, lo que existe, no es, sino, una promesa de futuro, con la calma en su espera, orientada a un problema de simple gestión pragmática de aplicación -hasta la excusa de falta de recursos-. De ahí que, la única forma posible de proyectarlo, es lidiar con la comunidad científica docente y enfrentar esta dicotomía o dualidad, que en algún sentido, mantiene el statu quo y que se manifiesta como una contradicción objetiva, que tensa por un lado entre los requerimiento de avance del ser social y el 
obstáculo que realizan las relaciones de poder, con el apoyo de modelos educativos o su aplicación insuficiente, en relación al proceso educativodocente que los conductores, e inclusive el docente del aula, no están dispuestos a ceder.

4. A nivel de sociedad, si bien, al demandar ésta sujetos formados acordes a las necesidades del mundo complejo, hay un riesgo de potenciar humanos críticos de éstas. Pero, las consecuencias de ello, es la producción de una tensión que se da por la disociación del sujeto y su mundo. Lo que, en última instancia hace, es formar jóvenes que se sienten ajenos a los procesos sociales, con un gran sello individualista y por otro, docentes con poco compromiso histórico.

5. Devela la incoherencia de las políticas públicas educacionales bajo el supuesto de entidades discretas divisibles entre educación y no educación -que, por cierto, como políticas, superan el ámbito de la escuela-, lo que provoca que el docente se sienta dueño de la esfera del proceso general de formación de conocimiento minusvalorando cualquier otro ámbito de información por falta de control - como una suerte de microfísica de poderes, como diría Foucault-, o bien se puede sostener que las existentes políticas públicas- son las que efectivamente quieren provocar aquello.

6 . Al negar que los procesos cognitivos contengan valor, no se reconoce, -contradictoriamente-, que el docente valora como hegemónico el conocimiento de la escuela, el suyo sobre otros. De ahí que toda valoración implique responsabilidad histórica.

7. Se produce la noción que separa al conocimiento de la realidad, al niño de su vida. No reconoce que al disociar la escuela de la calle, está valorando y por ende debe hacerse responsable de esta elección.

\section{Presencia del paradigma científico complejo}

Los modelos educacionales basados en el paradigma científico complejo, como el constructivismo, las propuestas de Morin ${ }^{5}$ o Lipman, entre otras, deben guardar coherencia con le nuevo cuadro científico de mundo. De ahí que el proceso de enseñanza-aprendizaje debe contemplar:

1- Que la dualidad cartesiana, es un pensamiento superado por la realidad. El sujeto actual se concibe ya sea como un micro sistema, como sistema autopoiético como lo acuñara Maturana, Varela y Uribe, de todas formas, no es un ladrillo, es una relación. Los alumnos no son objetos, y una conside-

\footnotetext{
${ }^{5}$ No hace falta explicitar su conocida propuesta, de todos modos, no se puede dejar de citar textos clásicos como Los siete saberes necesarios a la educación del futuro, Introducción al pensamiento complejo. El método, y tantos otros que enriquecen nuestra mirada.
} 
ración así, representa, como dijimos, una imposición de poder. Debe, por consiguiente, plantearse con ellos y como sujetos insertos en el tejido social.

2- La reconstrucción del sujeto, del conocimiento, del mundo y la vida como su lugar propio y no la entidad discreta-escuela.

3- Para este paradigma, los sistemas de ideas, teorías y conocimiento, es dinámico y emergente, dado lo cual, lo único posible por hacer, es permitir al alumno, construir el suyo, sin caer en un solipsismo o idealismo subjetivo, por eso que se da, a partir de su construcción contextuada dentro de una estructura social.

4- De acuerdo a lo anterior, resulta desfasada la creencia que permite sostener, la transmisión de un conocimiento.

5- La inexistencia de un sujeto-alumno-átomo-lineal, permite potenciar el pensamiento crítico. Pues, uno de los motivos de su no desarrollo, consiste, precisamente en las incoherencias epistemológicas del proceso de enseñanza-aprendizaje.

6- Ni la realidad que se pretende facilitar, a través, del proceso educativo, ni el proceso de enseñanza-aprendizaje, representan una dinámica causal, predictiva y por consiguiente, los desórdenes, la incertidumbre y el caos son parte integrantes del proceso y deben ser reconocidas. Lo anterior no implica el reconocimiento de un caos epistemológico, ni la imposibilidad de actuar con claridad en el mundo, ni, tampoco, una construcción subjetivada del mundo; por el contrario, implica la consideración a una perspectiva dialéctica, sólo que, se habían eliminado ciertos componentes, como las relaciones contradictorias, sus unidades, sus interconexiones lineales y no lineales, sus pasos adelante y atrás.

7- El aporte del Pensamiento Complejo mediante la aplicación de sus criterios o principios como lo son, el principio sistémico u organizacional, principio hologramático, principio de retroactividad, principio de recursividad, principio de autonomía/dependencia, principio dialógico y principio de reintroducción del cognoscente en todo conocimiento (Morin, 1999, 2003), posibilitan interrelacionar todas las separaciones del paradigma anterior.

8- Y la aceptación que todo proceso cognitivo contiene valoración, permite que el juez del conocimiento no sea el profesor, ni lo que enseña sea un dogma neutral, sino que sea la propia práctica quien decida. Por lo mismo, la responsabilidad, como afirmáramos se hace histórica. 


\section{El todo integrado (interpretación): Una trama posible a proponer}

Nos permitimos plantear algunas ideas finales, para el debate.

El proceso educativo -en general- es dialéctico y por consiguiente, con mayor grado de énfasis en las dinámicas y relaciones que en las estructuras. En tal sentido consideramos que:

a) Del alumno como entidad discreta a un mínimo ecosocial concreto y contextualizado:

El alumno, debe ser considerado desde su perspectiva real y no como un abstracto-ideal; es decir, como un mínimo ecosocial concreto y contextualizado (Gómez 2001, 2007), de ahí que no sea un objeto o un sujeto desconectado. Por lo tanto, dicho de otra manera, implica reconocer que en él, confluye el proceso histórico-natural; en última instancia, determinado materialmente; y lo porta, como un mínimo concreto. Sentido que nos cambia todo el sentido del proceso educativo. En tanto, desde esta perspectiva, se relaciona con la totalidad, a través, del proceso de formación.

b) La educación como proceso único:

La educación, como proceso de formación de los sujetos como actores sociales -más aún comprendidos como mínimos ecosociales concretos y contextualizados-, obliga a admitir su indivisibilidad -educación formal e informal-. Es decir, no se puede concebir como una entidad discreta -la escuela- y su difuso no yo. Por lo tanto, fraccionar o reducir el proceso representa poder. De acuerdo a ello, las políticas públicas educacionales, deben explicitarse de manera integral -o por nosotros visualizadas; lo que implica, la interrelación con las políticas económicas, sociales etc. Así por ejemplo, si la formación económico-social actual implica, sobrevivir en base a la competencia, no se puede esperar que los alumnos reproduzcan la solidaridad como valor, transmitida en el aula. Por lo tanto, una política pública de educación, cuando disocia esta unidad y se plantea reduccionistamente y no considera las relaciones y contradicciones en su totalidad, carece de veracidad, pues su discurso no es real, ni menos práctico.

c) La práctica como criterio de verdad.

Difícil resulta avalar una teoría, modelo pedagógico e incluso un paradigma o proceso de enseñanza-aprendizaje, desde la idea; ello es escolástico. ${ }^{6}$

\footnotetext{
${ }^{6}$ Compartimos el pensamiento de Marx sintetizado en la segunda Tesis sobre Feuerbach “ El problema de si al pensamiento humano se le puede atribuir una verdad objetiva, no es un problema teórico, sino un problema práctico. Es en la práctica donde el hombre tiene que demostrar la verdad, es decir, la realidad y el poderío, la terrenalidad de su pensamiento. El litigio sobre la realidad o irrealidad de un pensamiento que se aísla de la práctica, es un problema puramente escolástico”. (Marx, K. y Engels, 1955: 426)
} 
Es la práctica la que establece el criterio de verdad, de ahí que se imposibilita todo atisbo de conocimiento acabado y por consiguiente, a transmitir un constructivismo basado en el idealismo subjetivo; la primacía de la idea sobre la realidad; una categoría sin la mediación del sujeto-objeto histórico; como también la neutralidad y objetividad, en tanto en la práctica hay relaciones de poder que se reproducen.

En definitiva, todo es parte del mismo movimiento histórico y los sujetos, como actores sociales, deben ser formados para esta realidad compleja-; no obstante, no obviamos las diferencias que tienen éstos en las distintas posiciones prácticas en las que participan; por lo tanto, el proceso de enseñanza-aprendizaje, tiene una connotación adscrita a los modos de producción, a los cuales, acceden y en ello retomamos las reflexiones sobre la educación realizadas, hace más de un siglo, por el clásico Aníbal Ponce (1972).

\section{Conclusiones}

La formación económico-social, determina los distintos cuadros de mundos que se producen en ella.

El cuadro científico clásico de mundo, produjo un conocimiento científico basado, en lo que ahora denominamos, paradigma científico clásico.

El -contextuado en el cuadro científico clásico de mundo- hizo coherente un determinado modelo educativo, en tanto, el requerimiento del ser ecosocial de humanos para interactuar en él, de acuerdo a las contradicciones prácticas del proceso del trabajo.

Los cambios en las dinámicas del trabajo cada vez más universal y contradictorios, dieron paso a un nuevo paradigma científico complejo, de mayor coherencia con el cuadro de mundo actual. De ahí que, los modelos educacionales, fueran propuestas coherentes.

La tensión entre los modelos ideales nuevos e históricamente necesarios y las relaciones de poder que mantienen la aplicación de modelos clásicos, a pesar que ello no se visualiza, provocan distorsiones que, en definitiva ocasionan alienación.

La superación será el resultado de la agudización de esta crisis.

Hay un salto cualitativo que no se puede obviar y que distingue una actitud reformadora de una revolucionaria; pues no sólo se requiere, de acuerdo a la primera, sujetos formados en el paradigma científico complejo como actores para un mundo complejo; es decir, no sólo debemos formar sujetos que entiendan el mundo en que viven, sino que, además lo transformen. 


\section{Bibliografía}

Bacon, F. (1998), Teoría del cielo. Ediciones Altaza, Barcelona.

Delgado, C. (1999), “El cambio de racionalidad y la matematización del saber”, artículo publicado en la Revista colombiana de filosofía de la ciencia Vol. $N^{\circ} 1$. Colombia.

Delgado, C. (2004), La importancia política de las cosas pequeñas (Ponencia presentada en el Panel de Clausura del Evento Complejidad, La Habana. (paper).

Gómez T. (2001), La Contradicción final. (Ponencia presentada en la Conferencia Internacional de Derecho Ambiental, Pinar del Río, Cuba (14-17, marzo), (paper).

Idem (2007), La conciencia ecológica: una nueva forma de la conciencia social, Tesis de Doctor en Ciencias Filosóficas, no publicada. Universidad de La Habana, Cuba.

Idem. (2003), Obstáculos para que el constructivismo se realice en nuestro país, Tesina de Diplomado Enfoque contemporáneo del proceso docente-educativo, no publicada. Santiago de Chile 2003.

Kedrov, B., (1990), Lenin y las revoluciones científicas, Editorial de Ciencias Sociales, La Habana.

Lipman, M. (1998), Pensamiento Complejo y educación, Ediciones de La Torre, Madrid.

Maldonado, C. (Ed.). (1999), Visiones sobre la complejidad. Ediciones El Bosque, Bogotá.

Marx, K. y Engels, F. (1955), “Tesis sobre Feuerbach”, en Obras escogidas en dos tomos: Tomo II, Ediciones en lenguas extranjeras Moscú.

Morin, E (1999), Los siete saberes necesarios a la educación del futuro, Organización de las Naciones Unidas para la Educación, la Ciencia y la Cultura. UNESCO, Paris.

Idem. (2003), Introducción al pensamiento complejo, Gedisa, España.

Pérez, C. (1996), Sobre la condición social de la psicología, LOM, Santiago de Chile.

Ponce, A. (1972), Educación y lucha de clases, Editorial Nascimiento, Santiago de Chile.

Sotolongo, L y Delgado, C. (2006), La revolución contemporánea del sa- 
Polis, Revista de la Universidad Bolivariana, Volumen 9, $N^{\circ}$ 25, 2010

ber y la complejidad social: Hacia unas ciencias sociales de nuevo tipo. Recuperado el 5 de Diciembre de 2008 del sitio Web de Clacso. http:// bibliotecavirtual.clacso.org.ar/ar/libros/campus/soto/soto.html. 